\title{
Intrinsic electromechanical dynamic equations for piezoelectric power harvesters
}

\begin{abstract}
This paper discusses, compares and contrasts two important techniques for formulating the electromechanical piezoelectric equations for power harvesting system applications. It presents important additions to existing literature by providing intrinsic formulation techniques of the harvesting system for the two different electromechanical dynamic equation-based voltage and charge type systems associated with the standard AC-DC circuit interface developed using the extended Hamiltonian principle. The derivations of the two analytical methods rely on the fundamental continuum thermopiezoelectricity concepts of the electrical enthalpy energy and Helmholtz free energy. The benefit of using analytical charge type modelling is that the technique shows more compact formulation for developing simultaneous derivations by coupling the mechanical and electromechanical systems of the piezoelectric devices and electronic system so that the frequency response functions (FRFs) and time wave form systems can be formulated. On the other hand, the analytical voltage type modelling is obviously convenient but can show tedious derivation process for joining with the electronic circuit part. To tackle this situation, the analytical voltage type with mechanical and electromechanical forms of the piezoelectric structure can be derived separately from the electronic system where they can be combined together after applying further derivations to formulate the FRFs. In this paper, the two analytical techniques also show particular benefit and even further development of how to model the power harvesting scheme with the combinations of piezoelectric structure and electronic system. Moreover, validations of the two analytical methods show good agreement with the previous authors' electromechanical finite element analysis and experimental works. Further parametric electromechanical energy harvesting behaviours have been explored to study the system responses.
\end{abstract}

Keywords: Charge and voltage type-based electromechanical equations - Energy harvesting . Electromechanics · Frequency and time waveform responses · Piezoelectric · Vibration.

\section{Introduction}

Robust micro-power harvesters provide capabilities for capturing the surrounding vibration environment and converting it into usable electric energy that can be further processed via electronic management systems for powering autonomous wireless sensor devices. The increasing technical challenge for investigating piezoelectric systems requires essential mathematical studies in many different applications. In the earlier studies, the application of the piezoelectric structures have been derived theoretically for formulating shape control for sensing and actuation under static and dynamic systems [1]-[2] and thermal expansion systems [3], the feedback gain control systembased sensor and actuator systems [4]-[6], and shunt control systems [7]-[8]. 
The vast majority of smart structure power harvesters using different theoretical studies have been focused on the piezoelectric devices with different geometry, physical property and parametric case studies. Most notably power harvesting structures under base excitation for generating significant electrical output have included the typical cantilevered piezoelectric laminates (unimorph and bimorph) either with or without proof mass using electromechanical lumped parameter models with electrical equivalent system [9], analytical approach techniques [10]-[14] modal analysis method [15], closed form boundary value techniques [16] and electromechanical finite element analysis [17]-[20]. Another alternative approach for widening the frequency bandwidth [21]-[26] has been investigated using multiple piezoelectric beams representing an improved power harvester system level so as to adapt with the ambient vibration environment that changes over time resulting in different resonances.

Focusing on the technical DC harvesting circuit interfaces has become crucial to the studies to optimize the power output. The majority of these have considered the integrated electronic systems with the piezoelectric structures investigated using SPICE software with the use of equivalent circuit parameter identification-based ANSYS simulation [18], analytical modal analysis-based DC interface [15], SCE (synchronized charge extraction) technique [27]-[28], and analytical standard parallel-SSHI and series-SSHI (synchronized switch harvesting on inductor) interfaces [27], [29]-[30]. New SSHI architecture using replacement of the other two diodes with the two separated detection switches were also be developed for maximizing power output with the limited losses from voltage gaps [31]. Recently, the new analytical equivalent circuit systems-based SSHI techniques [19] were developed for identifying the system parameters for use in the COMSOL finite element simulation. Moreover, the new analytical studies for self-adaptive DC power optimization [32] has been developed using the shunt circuit control techniques on the piezoelectric layer for tuning the other piezoelectric layer for the electrical energy extraction. More importantly, the parametric circuit identifications were used to model the appearance of the two simultaneous resonance frequencies at the first mode using only a single piezoelectric bimorph with proof mass offset.

The primary purpose to present this paper is to provide the technical insight and concepts of intrinsic electromechanical system formulations of piezoelectric power harvester with proof mass offset. The techniques show the explorations of key equations using two different analytical charge and voltage type methods with circuit interface. At this stage, there are no previous theoretical works presenting the intrinsic formulation techniques in relations to the piezoelectric system and the harvesting circuit for formulating the power harvesting frequency and time waveform responses. The two methods reduced from the fundamental thermopiezoelectricity concepts and dynamical closed-form boundary value equations were developed using the extended Hamiltonian principle. It can be reasonably expected to express the intrinsic physical properties for the use of the two different methods coupled with the electronic system. It was found that the charge type system provides a direct method of solving the simultaneous electromechanical equations using the functional energy forms representing a compact system modelling approach compared with the voltage type system that still requires the derivation separately from the electronic system. Moreover, frequency and time waveform response systems with the standard AC-DC circuit interface can also be included. For complementary studies, the validations of the two analytical methods were carried out and compared with the established electromechanical finite element vibration and experimental results, giving good agreement. Further parametric case studies under variable resistance and capacitance including DC time waveform electrical output behaviours were also discussed.

\section{Electromechanical Constitutive Relations}

In Fig. 1, the typical example of the piezoelectric unimorph smart structure is shown with proof mass offset under base excitation and connected with the standard AC-DC harvesting circuit. At the next stage, the key equations for modelling the system are presented to formulate the analytical charge and voltage type systems. 


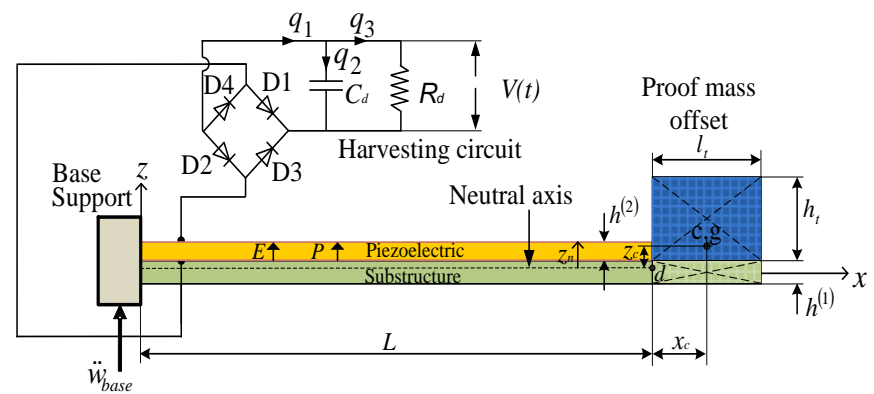

Fig. 1 Physical system of power harvesting piezoelectric beam with dynamical proof mass offse under base input excitation.

\subsection{Voltage type-based constitutive electromechanical formulations}

The constitutive piezoelectric equations of the second layer based on the stress-electric displacement relation in terms of 3-1 mode of piezoelectric operation and 3-3 effect of the permittivity can be formulated as,

$$
\begin{aligned}
& T_{1}^{(2)}=\bar{c}_{11}^{(2, E)} S_{1}^{(2)}-e_{31}^{(2)} E_{3}^{(2)}, \\
& D_{3}^{(2)}=e_{31}^{(2)} S_{1}^{(2)}+\varepsilon_{33}^{(2, S)} E_{3}^{(2)},
\end{aligned}
$$

where, $\quad \varepsilon_{33}^{(2, S)}=\varepsilon_{33}^{(2, T)}-d_{31}^{2} \bar{c}_{11}^{(2, E)}, e_{31}=d_{31} \bar{c}_{11}^{E}$,

$$
E_{3}=-\nabla \varphi(z, t)=-v(t)(\mathrm{d} \vartheta(z) / \mathrm{d} z), \quad \vartheta(z, t)=\left(z-z_{n}+h^{(2)}\right) / h^{(2)} .
$$

The general strain field for each layer and stress field of the substructure can be stated respectively as,

$$
S_{1}(x, t)=-z \frac{\partial^{2} w(x, t)}{\partial x^{2}}, \quad T_{1}^{1}=\bar{c}_{11}^{(1)} S_{1}^{(1)} .
$$

Note that the notations of the piezoelectric material are written according to the IEEE standards [33]. Superscripts 1 and 2 indicate substructure and piezoelectric layers, respectively. The parameters $T, S, E, D$ and $\varphi$ represent stress, strain, electric field, electric displacement and electrical potential, respectively. Moreover, the coefficients $c, e, \varepsilon_{33}^{S}$ and $\vartheta$ indicate elastic constant, piezoelectric coefficient, permittivity at constant strain, and electrical shape function, respectively.

2.2 Charge type-based constitutive electromechanical formulations

The constitutive piezoelectric equations at the second layer based on stress-electric field relations can be formulated as,

$$
\begin{gathered}
T_{1}^{(2)}=\bar{c}_{D}^{(2)} S_{1}^{(2)}-g_{31}^{(2)} D_{3}^{(2)}, \\
E_{3}^{(2)}=-g_{31}^{(2)} S_{1}^{(2)}+\varepsilon_{33}^{(2, S)^{-1}} D_{3}^{(2)},
\end{gathered}
$$

where, $\quad \bar{c}_{D}^{(2)}=\bar{c}_{11}^{(2, E)}+e_{31}^{(2)^{2}} \varepsilon_{33}^{(2, S)^{-1}}, g_{31}^{(2)}=\varepsilon_{33}^{(2, S)^{-1}} e_{31}^{(2)}$.

\section{Variational Energy Expressions}

\subsection{Mechanical system-based energy expressions}

The kinetic energy for the unimorph structure with proof mass offset $\left(K E=K E^{(1)}+K E^{(2)}+K E^{t i p}\right)$ can be formulated as,

$$
\begin{aligned}
K E=\frac{1}{2} \int_{0}^{L} \int_{A^{(1)}} \rho^{(1)} \dot{w}(x, t)^{2} \mathrm{~d} A^{(1)} \mathrm{d} x+\frac{1}{2} \int_{0}^{L} \int_{A^{(2)}} \rho^{(2)} \dot{w}(x, t)^{2} \mathrm{~d} A^{(2)} \mathrm{d} x \\
+I_{0}^{t i p} x_{c} \dot{w}(L, t) \dot{\theta}(L, t)+\frac{1}{2} I_{0}^{t i p} \dot{w}(L, t)^{2}+\frac{1}{2} I_{2}^{t i p} \dot{\theta}(L, t)^{2} .
\end{aligned}
$$


Note that details of the kinetic energy equations including the parameters of the proof mass offset $I_{0}^{t i p}$ and $I_{2}^{t i p}$ can be seen in [20]. The non-conservative work due to the input base excitation $\left(W F=W F^{(1)}+W F^{(2)}+W F^{\text {base }}\right)$ can be formulated as,

$$
\begin{aligned}
W F= & -\int_{0}^{L} \int_{A^{(1)}} \rho^{(1)} w(x, t) \mathrm{d} A^{(1)} \mathrm{d} x \ddot{w}_{\text {base }}(t)-\int_{0}^{L} \int_{A^{(2)}} \rho^{(2)} w(x, t) \mathrm{d} A^{(2)} \mathrm{d} x \ddot{w}_{\text {base }}(t) \\
& -I_{0}^{t i p} x_{c} \theta(L, t) \ddot{w}_{\text {base }}(t)-I_{0}^{t i p} w(L, t) \ddot{w}_{\text {base }}(t) .
\end{aligned}
$$

Note that details of Eq. (5) can be found in [20].

\subsection{Voltage type-based energy expressions}

The potential energy of the unimorph structure $\left(P E=P E^{(1)}+P E^{(2)}\right)$ can be formulated using (1a) and (2) to give,

$$
\begin{aligned}
P E & =\frac{1}{2} \int_{0}^{L} \int_{A^{(1)}} S_{1}^{(1)} T_{1}^{(1)} \mathrm{d} A^{(1)} \mathrm{d} x+\frac{1}{2} \int_{0}^{L} \int_{A^{(2)}} S_{1}^{(2)} T_{1}^{(2)} \mathrm{d} A^{(2)} \mathrm{d} x \\
& =\frac{1}{2} \int_{0}^{L} \int_{A^{(1)}} \bar{c}_{11}^{(1)} S_{1}^{(1)^{2}} \mathrm{~d} A^{(1)} \mathrm{d} x+\frac{1}{2} \int_{0}^{L} \int_{A^{(2)}} \bar{c}_{11}^{(2)} S_{1}^{(2)^{2}} \mathrm{~d} A^{(2)} \mathrm{d} x-\frac{1}{2} \int_{0}^{L} \int_{A^{(2)}} e_{31}^{(2)} E_{3}^{(2)} S_{1}^{(2)} \mathrm{d} A^{(2)} \mathrm{d} x .
\end{aligned}
$$

The electrical energy of the piezoelectric element $\left(W E=W E^{(2)}\right)$ using (1b) can be stated as,

$$
\begin{aligned}
W E & =\frac{1}{2} \int_{0}^{L} \int_{A^{(2)}} E_{3}^{(2)} D_{3}^{(2)} \mathrm{d} A^{(2)} \mathrm{d} x, \\
& =\frac{1}{2} \int_{0}^{L} \int_{A^{(2)}} e_{31}^{(2)} S_{1}^{(2)} E_{3}^{(2)} \mathrm{d} A^{(2)} \mathrm{d} x+\frac{1}{2} \int_{0}^{L} \int_{A^{(2)}} \varepsilon_{33}^{(2, S)} E_{3}^{(2)^{2}} \mathrm{~d} A^{(2)} \mathrm{d} x .
\end{aligned}
$$

\subsection{Charge type-based energy expressions}

The potential energy of the unimorph structure $\left(P E^{*}=P E^{(1)^{*}}+P E^{(2)^{*}}\right)$ using (2) and (3a) can be stated as,

$$
P E^{*}=\frac{1}{2} \int_{0}^{L} \int_{A^{(1)}} \bar{c}_{D}^{(1)} S_{1}^{(1)^{2}} \mathrm{~d} A^{(1)} \mathrm{d} x+\frac{1}{2} \int_{0}^{L} \int_{A^{(2)}} \bar{c}_{D}^{(2)} S_{1}^{(2)^{2}} \mathrm{~d} A^{(2)} \mathrm{d} x-\frac{1}{2} \int_{0}^{L} \int_{A^{(2)}} g_{31}^{(2)} D_{3}^{(2)} S_{1}^{(2)} \mathrm{d} A^{(2)} \mathrm{d} x .
$$

The electrical energy of the piezoelectric element $\left(W E^{*}=W E^{(2)^{*}}\right)$ using (3b) can be stated as,

$$
W E^{*}=-\frac{1}{2} \int_{0}^{L} \int_{A^{(2)}} g_{31}^{(2)} S_{1}^{(2)} D_{3}^{(2)} \mathrm{d} A^{(2)} \mathrm{d} x+\frac{1}{2} \int_{0}^{L} \int_{A^{(2)}} \varepsilon_{33}^{(2, S)^{-1}} D_{3}^{(2)^{2}} \mathrm{~d} A^{(2)} \mathrm{d} x .
$$

The electrical energy of the capacitor can be formulated as,

$$
W C=\frac{1}{2 C_{d}} q_{2}(t)^{2} .
$$

The electrical work dissipated by the resistor can be stated as,

$$
\delta W R=-R_{d} \dot{q}_{3}(t) \delta q_{3}(t)
$$

\subsection{Distinct voltage- and charge-type piezoelectric energy expressions}

There are distinct procedures for applying the functional energy expressions of voltage and charge type piezoelectric system equations. The fundamental techniques of the piezoelectric energy expressions rely on the continuum thermopiezoelectricity concepts that provide direct relevance to the power harvesting application. They provide the technical basis for formulating voltage- and charge-type Hamiltonian principle as shown in the next sections. Here, we introduce the brief technical concepts.

For the voltage-type piezoelectric energy expression, it can be proved using linear electrical enthalpy of the piezoelectric material in the tensor notation where it can be formulated according 
to the continuum thermodynamics which can be condensed using Voigt's notation and then further reduced using Einstein's summation convention [34]-[35] as,

$$
H\left(S_{1}^{(2)}, E_{3}^{(2)}\right)=\frac{1}{2} \bar{c}_{11}^{(2, E)} S_{1}^{(2)^{2}}-\mathrm{e}_{31}^{(2)} S_{1}^{(2)} E_{3}^{(2)}-\frac{1}{2} \varepsilon_{33}^{(2, S)} E_{3}^{(2)^{2}} .
$$

Note that the electrical enthalpy under adiabatic and isothermal processes is obviously used in many power harvesting applications. Here, the electrical enthalpy can also be formulated as,

$$
H\left(S_{1}^{(2)}, E_{3}^{(2)}\right)=\frac{1}{2} \mathrm{~T}_{1}^{(2)} S_{1}^{(2)}-\frac{1}{2} E_{3}^{(2)} D_{3}^{(2)} .
$$

Substituting (1) into (13) gives,

$$
H\left(S_{1}^{(2)}, E_{3}^{(2)}\right)=\frac{1}{2}\left(\bar{c}_{11}^{(2, E)} S_{1}^{(2)}-\mathrm{e}_{31} \mathrm{E}_{3}\right) S_{1}^{(2)}-\frac{1}{2}\left(\mathrm{e}_{31}^{(2)} S_{1}^{(2)}+\varepsilon_{33}^{(2, S)} E_{3}^{(2)}\right) E_{3}^{(2)} .
$$

Then, it gives the following electrical enthalpy expression with similar form with (12). Eq. (13) can also be formulated using the exact differential form as,

$$
\delta H\left(S_{1}^{(2)}, E_{3}^{(2)}\right)=T_{1}^{(2)} \delta S_{1}^{(2)}-D_{3}^{(2)} \delta E_{3}^{(2)}=\delta P E^{(2)}-\delta W E^{(2)} .
$$

For the charge-type piezoelectric energy expression, the Helmholtz free energy under adiabatic and isothermal processes [36]-[37] can be used for conceptualising the charge type as,

$$
F\left(S_{1}^{(2)}, D_{3}^{(2)}\right)=\frac{1}{2} c_{D}^{(2)} S_{1}^{(2)^{2}}-\mathrm{g}_{31}^{(2)} S_{1}^{(2)} D_{3}^{(2)}+\frac{1}{2} \varepsilon_{33}^{(2, S)^{-1}} D_{3}^{(2)^{2}} .
$$

The fundamental Helmholtz free energy can also be formulated as,

$$
F\left(S_{1}^{(2)}, D_{3}^{(2)}\right)=\frac{1}{2} T_{1}^{(2)} S_{1}^{(2)}+\frac{1}{2} E_{3}^{(2)} D_{3}^{(2)} .
$$

Substituting (3) into (17) gives,

$$
F\left(S_{1}^{(2)}, D_{3}^{(2)}\right)=\frac{1}{2}\left(c_{D}^{(2)} S_{1}^{(2)}-g_{31}^{(2)} D_{3}^{(2)}\right) S_{1}^{(2)}+\frac{1}{2}\left(-g_{31}^{(2)} S_{1}^{(2)}+\varepsilon_{33}^{(2, S)^{-1}} D_{3}^{(2)}\right) D_{3}^{(2)} .
$$

Thus, Eq. (18) can be seen to have similar form with (16). Eq. (17) can also be formulated using the exact differential form as,

$$
\delta F\left(S_{1}^{(2)}, D_{3}^{(2)}\right)=T_{1}^{(2)} \delta S_{1}^{(2)}+E_{3}^{(2)} \delta D_{3}^{(2)}=\delta P E^{(2) *}+\delta W E^{(2)^{*}} .
$$

It should be noted here that the expressions of the voltage- and charge-type piezoelectric energy expressions can be seen in the technical concept for understanding the functional energy forms using electrical enthalpy energy and Helmholtz free energy. The differences can be found not only in the sign form (negative sign $-\delta W E^{(2)}$ in $(15)$ and positive sign $+\delta W E^{(2) *}$ in (19)) but also in the corresponding coefficients from (1) and (3). However, when including these expressions into Hamiltonian principle as shown in Sections 4.1 and 4.2, the expressions become positive sign $+\delta W E^{(2)}$ and negative $\operatorname{sign}-\delta W E^{(2) *}$. Note that, as shown previously, the expressions $\left(\delta W E=\delta W E^{(2)}\right)$ and $\left(\delta W E^{*}=\delta W E^{(2) *}\right)$ represent piezoelectric electrical energies for voltage- and charge-type systems, respectively.

\section{Electromechanical closed-form boundary value method}

This section discusses the two analytical methods using the extended Hamiltonian principle to formulate the normalised closed-form electromechanical transverse dynamic equations. To understand the idea of the technical concepts of the paper, the analytical charge type modelling is discussed first followed by analytical voltage type modelling.

\subsection{Charge type-based electromechanical equations}

\subsubsection{Non-rectified piezoelectric harvesting circuit}

This section discusses key equations of derivation for the piezoelectric energy harvesters with tip mass offset that can be formulated using the extended Hamiltonian principle by including circuit harvesting and non-conservative work to give, 


$$
\left.\begin{array}{l}
\int_{t_{1}}^{t_{2}} \delta\left(L_{a}+W_{f}\right) d t=0
\end{array}\right\} \begin{gathered}
L_{a} \in\left\{K E, P E^{*}, W E^{*}, W C\right\} \\
W_{f} \in\{W F, W R\}
\end{gathered} \text { or }
$$

After substituting Eq. (19) into Eq. (20) and further using the expression $\left(P E^{*}=P E^{(1)^{*}}+P E^{(2)^{*}}\right)$, Eq. (20) can be reformulated as,

$$
\int_{t_{1}}^{t_{2}}\left(\delta K E-\delta P E^{*}-\delta W E^{*}+\delta W F-\delta W C+\delta W R\right) \mathrm{d} t=0 .
$$

Note that each functional energy term of (21) can be formulated in (4)-(5) and (8)-(11). Also, as discussed previously, the expression $-\delta W E^{*}$ can be seen in the charge-type system. The functional forms $L_{a}$ and $W_{f}$ from Hamiltonian's principle can be seen as the continuous differentiable functions of virtual displacement, electric displacement and charge for the whole system that can be stated as,

$$
\begin{aligned}
& L_{a}=L_{a}\left(\dot{w}(x, t), \dot{w}(L, t), \frac{\partial \dot{w}(L, t)}{\partial x}, \frac{\partial^{2} w(x, t)}{\partial x^{2}}, D_{3}^{(2)}(z, t), q_{2}(t)\right), \\
& W_{f}=W_{f}\left(w(x, t), \frac{\partial w(L, t)}{\partial x}, w(L, t), q_{3}(t)\right) .
\end{aligned}
$$

Eq. (22) can be further formulated using total differential equations as,

$$
\begin{aligned}
\delta L_{a}= & \frac{\partial L_{a}}{\partial \dot{w}(x, t)} \delta \dot{w}(x, t)+\frac{\partial L_{a}}{\partial \dot{w}(L, t)} \delta \dot{w}(L, t)+\frac{\partial L_{a}}{\partial\left(\frac{\partial \dot{w}}{\partial x}(L, t)\right)} \delta\left(\frac{\partial \dot{w}}{\partial x}(L, t)\right) \\
& +\frac{\partial L_{a}}{\partial\left(\frac{\partial^{2} w(x, t)}{\partial x^{2}}\right)} \delta\left(\frac{\partial^{2} w(x, t)}{\partial x^{2}}\right)+\frac{\partial L_{a}}{\partial D_{3}^{(2)}(z, t)} \delta D_{3}^{(2)}(z, t)+\frac{\partial L_{a}}{\partial q_{2}(t)} \delta q_{2}(t), \\
\delta W_{f} & =\frac{\partial W_{f}}{\partial w(x, t)} \delta w(x, t)+\frac{\partial W_{f}}{\partial\left(\frac{\partial w}{\partial x}(L, t)\right)} \delta\left(\frac{\partial w}{\partial x}(L, t)\right)+\frac{\partial W_{f}}{\partial w(L)} \delta w(L, t)+\frac{\partial W_{f}}{\partial q_{3}(t)} \delta q_{3}(t) .
\end{aligned}
$$

Corresponding with (23), Eq. (21) can be further formulated by using integro-differential equations and the variational principle to give the analytical coupled equation of the electrical shunted control and electromechanical power harvester system for the dynamical closed-form boundary value equation,

$$
\begin{aligned}
& \int_{t_{2}}^{t_{2}}\left[\int_{0}^{L}\left\{-I_{0} \ddot{w}(x, t)-I_{0} \ddot{w}_{\text {base }}(t)-C_{t} \frac{\partial^{2}}{\partial x^{2}}\left(\frac{\partial^{2} w(x, t)}{\partial x^{2}}\right)\right\} \delta w(x, t) \mathrm{d} x\right. \\
& -\left\{x_{c} I_{0}^{t i p} \frac{\partial \ddot{w}(L, t)}{\partial x}+I_{0}^{t i p} \ddot{w}(L, t)+I_{0}^{t i p} \ddot{w}_{\text {base }}\right\} \delta w(L, t)+\left.C_{t} \frac{\partial}{\partial x}\left(\frac{\partial^{2} w(x, t)}{\partial x^{2}}\right) \delta w(x, t)\right|_{0} ^{L} \\
& -\left\{I_{2}^{t i p} \frac{\partial \ddot{w}(L, t)}{\partial x}+x_{c} I_{0}^{(t i p)} \ddot{w}_{\text {base }}+x_{c} I_{0}^{t i p} \ddot{w}(L, t)\right\} \delta \frac{\partial w(L, t)}{\partial x} \\
& -\left.\left\{\eta q_{1}+C_{t} \frac{\partial^{2} w(x, t)}{\partial x^{2}}\right\} \delta \frac{\partial w(x, t)}{\partial x}\right|_{0} ^{L}-\left\{\int_{0}^{L} \eta \frac{\partial^{2} w(x, t)}{\partial x^{2}} d x+\frac{q_{1}(t)}{C_{v}}\right\} \delta q_{1}(t) \\
& \left.-\frac{q_{2}(t) \delta q_{2}(t)}{C_{d}}-R_{d} \dot{q}_{3}(t) \delta q_{3}(t)\right] \mathrm{d} t=0 .
\end{aligned}
$$

Note that parameter $D_{3}$ implied in Eq. (24) has been modified using $D_{3}=q_{1} / b L$. Each coefficient shown in Eq. (24) can be found in Appendices A, B and C and mass moments of inertia for unimorph $\left(I_{0}\right)$ and tip mass offset $\left(I_{0}^{t i p}\right.$ and $\left.I_{2}^{t i p}\right)$ can be seen in [20]. Applying KCL for the harvesting circuit in Fig. 1 gives, 


$$
q_{1}=q_{2}+q_{3} .
$$

As shown in Eq. (24), variable $q_{2}$ can be eliminated in the forthcoming reduced equations for simplicity using the relation as,

$$
\frac{q_{2}(t)}{C_{d}} \delta q_{2}(t)=\frac{q_{1}(t)}{C_{d}} \delta q_{1}(t)-\frac{q_{3}(t)}{C_{d}} \delta q_{1}(t)-\frac{q_{1}(t)}{C_{d}} \delta q_{3}(t)+\frac{q_{3}(t)}{C_{d}} \delta q_{3}(t) .
$$

After applying the mathematical lemma of duBois-Reymond's theorem for each virtual displacement field, the first constitutive electromechanical dynamic equation reduced from (24) can be formulated as,

$$
\delta w(x, t): \quad I_{0} \ddot{w}(x, t)+I_{0} \ddot{w}_{\text {base }}(t)+C_{t} \frac{\partial^{2}}{\partial x^{2}}\left(\frac{\partial^{2} w(x, t)}{\partial x^{2}}\right)=0 .
$$

The second and third constitutive electromechanical dynamic equations related piezoelectric and harvesting circuits can be formulated respectively as,

$$
\begin{aligned}
& \delta q_{1}(t): \int_{0}^{L} \eta \frac{\partial^{2} w(x, t)}{\partial x^{2}} \mathrm{~d} x+\left(\frac{1}{C_{v}}+\frac{1}{C_{d}}\right) q_{1}(t)-\frac{q_{3}(t)}{C_{d}}=0, \\
& \delta q_{3}(t): R_{d} \dot{q}_{3}(t)+\frac{q_{3}(t)}{C_{d}}-\frac{q_{1}(t)}{C_{d}}=0 .
\end{aligned}
$$

The boundary conditions can be formulated as,

$$
\begin{gathered}
w(0, t)=0 \quad, \frac{\partial w(0, t)}{\partial x}=0, \\
\delta w(L, t): x_{c} I_{0}^{t i p} \frac{\partial \ddot{w}(L, t)}{\partial x}+I_{0}^{t i p} \ddot{w}(L, t)+I_{0}^{t i p} \ddot{w}_{\text {base }}-C_{t} \frac{\partial}{\partial x}\left(\frac{\partial^{2} w(L, t)}{\partial x^{2}}\right)=0, \\
\delta \frac{\partial w(L, t)}{\partial x}: x_{c} I_{0}^{t i p} \ddot{w}_{b a s e}+x_{c} I_{0}^{t i p} \ddot{w}(L, t)+I_{2}^{t i p} \frac{\partial \ddot{w}(L, t)}{\partial x}+C_{t} \frac{\partial^{2} w(L, t)}{\partial x^{2}}+\eta q_{1}(t)=0 .
\end{gathered}
$$

The solution form of (27)-(29) can be formulated using mode superposition depending on the normalised mode shapes and generalised time dependent coordinates or the convergent eigenfunction forms to give,

$$
w(x, t)=\sum_{r=1}^{\infty} \hat{W}_{r}(x) w_{r}(t)
$$

The normalised mode shape can be found in [16]. The parameter $q_{1}(t)$ from (28a) associated with (30) can be substituted into (28b) to give,

$$
\sum_{r=1}^{\infty} \int_{0}^{L} \frac{\eta C_{v}}{\left(C_{v}+C\right)} \frac{\mathrm{d}^{2} \hat{W}_{r}(x)}{\mathrm{d} x^{2}} \mathrm{~d} x w_{r}(t)+\frac{1}{C_{d}}\left(1-\frac{C_{v}}{\left(C_{v}+C_{d}\right)}\right) q_{3}(t)+R_{d} \dot{q}_{3}(t)=0 .
$$

Further derivations can be achieved by several steps. First, Eq. (27) can be formulated by applying (30) and then multiplying with $\hat{W}_{q}(x)$. The result can then be integrated with respect to $x$. Second, Eq. (29a)-(29b) can be formulated by applying (30). After simplification, the closed-form electromechanical transverse dynamic equations under input base excitation can be formulated using the results obtained from the aforementioned steps associated with (31) to give,

$$
\begin{aligned}
& \int_{0}^{L} I_{0} \hat{W}_{r}(x) \hat{W}_{q}(x) \mathrm{d} x \ddot{w}_{r}(t)+I_{0}^{t i p} \hat{W}_{r}(L) \hat{W}_{q}(L) \ddot{w}_{r}(t) \\
& +x_{c} I_{0}^{t i p} \frac{\mathrm{d} \hat{W}_{r}(L)}{\mathrm{d} x} \hat{W}_{q}(L) \ddot{w}_{r}(t)+x_{c} I_{0}^{t i p} \hat{W}_{r}(L) \frac{\mathrm{d} \hat{W}_{q}(L)}{\mathrm{d} x} \ddot{w}_{r}(t) \\
& +I_{2}^{t i p} \frac{\mathrm{d} \hat{W}_{r}(L)}{\mathrm{d} x} \frac{\mathrm{d} \hat{W}_{q}(L)}{\mathrm{d} x} \ddot{w}_{r}(t)+\int_{0}^{L} C_{t} \frac{\mathrm{d}^{2} \hat{W}_{r}(x)}{\mathrm{d} x^{2}} \frac{\mathrm{d}^{2} \hat{W}_{q}(x)}{\mathrm{d} x^{2}} \mathrm{~d} x w_{r}(t) \\
& +\eta \frac{\mathrm{d} \hat{W}_{q}(L)}{\mathrm{d} x} q_{1}(t)=-\int_{0}^{L} I_{0} \hat{W}_{q}(x) \mathrm{d} \ddot{w}_{\text {base }}(t)-I_{0}^{t i p} \hat{W}_{q}(L) \ddot{w}_{\text {base }}(t)-x_{c} I_{0}^{t i p} \frac{\mathrm{d} \hat{W}_{q}(L)}{\mathrm{d} x} \ddot{w}_{\text {base }}(t) .
\end{aligned}
$$

Considering the orthonormality property of the mechanical dynamic equations from (32) gives,

$$
\int_{0}^{L} I_{0} \hat{W}_{r}(x) \hat{W}_{q}(x) \mathrm{d} x+I_{0}^{t i p} \hat{W}_{r}(L) \hat{W}_{q}(L)+x_{c} I_{0}^{t i p} \frac{\mathrm{d} \hat{W}_{r}(L)}{\mathrm{d} x} \hat{W}_{q}(L)
$$




$$
\begin{aligned}
& +x_{c} I_{0}^{t i p} \hat{W}_{r}(L) \frac{\mathrm{d} \hat{W}_{q}(L)}{\mathrm{d} x}+I_{2}^{t i p} \frac{\mathrm{d} \hat{W}_{r}(L)}{\mathrm{d} x} \frac{\mathrm{d} \hat{W}_{q}(L)}{\mathrm{d} x}=\delta_{r q}, \\
& \int_{0}^{L} C_{t} \frac{\mathrm{d}^{2} \hat{W}_{r}(x)}{\mathrm{d} x^{2}} \frac{\mathrm{d}^{2} \hat{W}_{q}(x)}{\mathrm{d} x^{2}} \mathrm{~d} x=\omega_{r}{ }^{2} \delta_{r q},
\end{aligned}
$$

where $\delta_{r q}$ is the Kronecker delta, defined as unity for $q=r$ and zero for $q \neq r$. Note that parameters $\hat{W}_{\mathrm{r}}(x)$ and $\hat{W}_{\mathrm{q}}(x)$ indicate normalised mode shapes. In terms of orthonormality, the Rayleigh mechanical damping can be formulated as,

$$
c_{r q}=\alpha \delta_{r q}+\beta \omega_{r}^{2} \delta_{r q}=2 \zeta_{r} \omega_{r} \delta_{r q} .
$$

where $\alpha$ and $\beta$ indicate Rayleigh damping coefficients. Corresponding to (33a)-(33b), Eq. (32) associated with (34) can now be reformulated to give the normalised closed-form electromechanical transverse dynamic equations,

$$
\ddot{w}_{r}(t)+2 \zeta_{r} \omega_{r} \dot{w}_{r}(t)+\omega_{r}^{2} w(t)+T_{r} q_{1}(t)=-Q_{r} \ddot{w}_{\text {base }}(t) .
$$

Eliminating $q_{1}(t)$ from (35) using (28a) associated with (30) gives,

$$
\begin{gathered}
\ddot{w}_{r}(t)+2 \zeta_{r} \omega_{r} \dot{w}_{r}(t)+\omega_{r}{ }^{2} w_{r}(t)-\sum_{r=1}^{\infty} \hat{T}_{r} T_{r} \gamma C_{d} w_{r}(t) \\
+T_{r} \gamma q_{3}(t)=-Q_{r} \ddot{w}_{\text {base }}(t) .
\end{gathered}
$$

Eq. (31) can be further simplified as,

$$
R_{d} \dot{q}_{3}(t)+P_{C d} q_{3}(t)+\sum_{r=1}^{\infty} \hat{T}_{r} \gamma w_{r}(t)=0 .
$$

At this case, the parameters $T_{r}, \hat{T}_{r}, P_{V}$, and $Q_{r}$ can be reduced as,

$$
\begin{gathered}
T_{r}=\eta \frac{\mathrm{d} \hat{W}_{r}}{\mathrm{~d} x}(L), \quad \sum_{r=1}^{\infty} \hat{T}_{r}^{(1)}=\sum_{r=1}^{\infty} \int_{0}^{L} \eta \frac{\mathrm{d}^{2} \hat{W}_{r}(x)}{\mathrm{d} x^{2}} \mathrm{~d} x, \\
P_{C d}=\frac{1}{C_{d}}(1-\gamma), \gamma=\frac{C_{v}}{\left(C_{v}+C_{d}\right)}, \\
Q_{r}=\int_{0}^{L} I_{0} \hat{W}_{r}(x) \mathrm{d} x+I_{0}^{t i p} \hat{W}_{r}(L)+x_{c} I_{0}^{t i p} \frac{\mathrm{d} \hat{W}_{r}(L)}{\mathrm{d} x} .
\end{gathered}
$$

To obtain the multi-mode electromechanical FRFs equations, Eqs. (36) and (37) can be further formulated using Laplace transformation giving the transfer functions which can be reduced into the frequency response relations. After simplification, the multimode electric charge FRFs can be formulated as,

$$
\frac{q_{3}(j \omega)}{-\omega^{2} w_{b a s e} e^{j \omega t}}=\frac{\sum_{r=1}^{m} \frac{Q_{r} \hat{T}_{r} \gamma}{\omega_{r}{ }^{2}-\hat{T}_{r} T_{r} \gamma C_{d}-\omega^{2}+j 2 \zeta_{r} \omega_{r} \omega}}{P_{C d}+j \omega R_{d}-\sum_{r=1}^{m} \frac{\hat{T}_{r} T_{r} \gamma^{2}}{\omega_{r}{ }^{2}-\hat{T}_{r} T_{r} \gamma C_{d}-\omega^{2}+j 2 \zeta_{r} \omega_{r} \omega}} .
$$

Other multimode FRFs relations can also be further formulated by using (39). Here, the multimode electric current FRFs can be formulated as,

$$
\frac{i_{3}(j \omega)}{-\omega^{2} w_{\text {base }} e^{j \omega t}}=j \omega \frac{q_{3}(j \omega)}{-\omega^{2} w_{\text {base }} e^{j \omega t}} .
$$

Note that since the capacitor and resistor are arranged in parallel connection, the voltage FRFs across the resistor or capacitor of the harvesting circuit can be formulated as,

$$
\frac{v(j \omega)}{-\omega^{2} w_{\text {base }} e^{j \omega t}}=j \omega R_{d} \frac{q_{3}(j \omega)}{-\omega^{2} w_{\text {base }} e^{j \omega t}}
$$

Power FRFs across the resistor and capacitor can be formulated respectively as,

$$
\begin{gathered}
\frac{P_{\text {Res }}(j \omega)}{\left(-\omega^{2} w_{\text {base }} e^{j \omega t}\right)^{2}}=-\omega^{2} R_{d} \frac{q_{3}(j \omega)^{2}}{\left(-\omega^{2} w_{\text {base }} e^{j \omega t}\right)^{2}}, \\
\frac{P_{\text {Cap }}(j \omega)}{\left(-\omega^{2} w_{\text {base }} e^{j \omega t}\right)^{2}}=-j \omega^{3} R_{d}{ }^{2} C_{d} \frac{q_{3}(j \omega)^{2}}{\left(-\omega^{2} w_{\text {base }} e^{j \omega t}\right)^{2}} .
\end{gathered}
$$




\subsubsection{DC piezoelectric harvesting circuit interface}

Fig. 2 shows the process of capturing AC electrical voltage from the piezoelectric element and converting it into DC voltage output via a full bridge rectifier and then further smoothed using a $\mathrm{RC}$ circuit. It can be seen that each time the capacitor conducts to charge, the DC current is generated. During the discharge time, the capacitor voltage decays and no current is supplied. This process repeats every half-cycle.

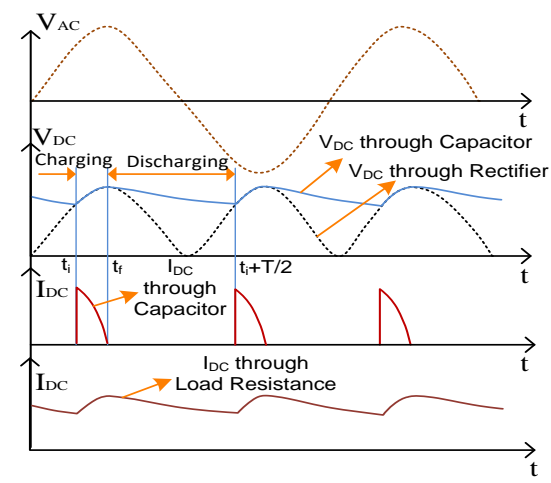

Fig. 2 Time waveform of standard harvesting circuit

a. Current flowing with interval $t_{i}<t<t_{f}$ indicating the charging time every half-cycle of the waveform.

For harvesting DC signal response, the AC-DC rectifier with smoothing RC circuit and piezoelectric system response can be formulated separately where the previous equation in (24) can be modified slightly by ignoring (10)-(11). Then, the new electrical work from the harvesting piezoelectric element $\delta W F_{r}=v_{d}(t) \delta q_{1}(t)$ is introduced into (21). The following equations of the coupled system response during the period of charging can be reformulated as,

$$
\begin{gathered}
\ddot{w}_{r}(t)+2 \zeta_{r} \omega_{r} \dot{w}_{r}(t)+\omega_{r}{ }^{2} w(t)+T_{r} q_{1}(t)=-Q_{r} \ddot{w}_{\text {base }}(t), \\
v_{d}+P_{V} q_{1}(t)+\sum_{r=1}^{\infty} \hat{T}_{r} w_{r}(t)=0,
\end{gathered}
$$

where $P_{V}=1 / C_{v}$. Differentiating (43b) with respect to time gives,

$$
\dot{v}_{d}+P_{V} \dot{q}_{1}(t)+\sum_{r=1}^{\infty} \hat{T}_{r} \dot{w}_{r}(t)=0 .
$$

The harvesting DC circuit equation can be formulated as,

$$
\dot{q}_{1}(t)-C_{d} \dot{v}_{d}-\frac{v_{d}}{R_{d}}=0 .
$$

Substituting parameter $q_{1}(t)$ from (43b) into (43a) and parameter $\dot{q}_{1}(t)$ from (45) into (44), the results of which can be formulated to give the following state space representation for the multimode response system as, 


$$
\frac{\mathrm{d}}{\mathrm{d} t}\left\{\begin{array}{c}
\dot{w}_{1} \\
\dot{w}_{2} \\
\cdot \\
w_{r} \\
\dot{w}_{1} \\
\dot{w}_{2} \\
\cdot \\
\cdot \\
\dot{w}_{r} \\
w_{2} \\
v_{d}
\end{array}\right\}=\left[\begin{array}{c}
\dot{w}_{r} \\
-2 \zeta_{1} \omega_{1} \dot{w}_{1}-\omega_{1}{ }^{2} w_{1}+\frac{\sum_{\mathrm{r}=1}^{\infty} \hat{T}_{\mathrm{r}} T_{\mathrm{r}} w_{r}}{P_{V}}+\frac{T_{1} v_{d}}{P_{V}}-Q_{1} \ddot{w}_{\text {base }}(t) \\
-2 \zeta_{2} \omega_{2} \dot{w}_{2}-\omega_{2}{ }^{2} w_{2}+\frac{\sum_{\mathrm{r}=1}^{\infty} \hat{T}_{\mathrm{r}} T_{\mathrm{r}} w_{r}}{P_{V}}+\frac{T_{2} v_{d}}{P_{V}}-Q_{2} \ddot{w}_{\text {base }}(t) \\
-2 \zeta_{\mathrm{r}} \omega_{\mathrm{r}} \dot{w}_{r}-\omega_{\mathrm{r}}{ }^{2} w_{r}+\frac{\sum_{\mathrm{r}=1}^{\infty} \hat{T}_{\mathrm{r}} T_{\mathrm{r}} w_{r}}{P_{V}}+\frac{T_{r} v_{d}}{P_{V}}-Q_{\mathrm{r}} \ddot{w}_{\text {base }}(t) \\
\sum_{\mathrm{r}=1}^{\infty} \hat{T}_{\mathrm{r}} \dot{w}_{r} \\
\left.P_{V} C_{d}+1\right)
\end{array}\right]
$$

b. Current flowing with interval $t_{f}<t<t_{i}+T / 2$ indicating the discharging times every halfcycle of the waveform.

At this case, the harvesting circuit can be formulated as,

$$
C_{d} \dot{v}_{d}+\frac{v_{d}}{R_{d}}=0
$$

The solution form of (47) can be stated as,

$$
v_{d}(t)=v_{d}\left(t_{f}\right) \exp \left(\frac{-\left(t-t_{f}\right)}{C_{d} R_{d}}\right) .
$$

Note that the expressions (46) and (48) can be utilized to estimate the current and voltage waveforms during the process of charging and discharging.

4.2 Voltage type-based electromechanical equations for non-rectifier harvesting circuit

This section discusses the key equations for the analytical voltage type-based piezoelectric energy harvesters. It should be noted that complete equations of this technique with only resistive loads can be seen in [16]. However, the extended technical aspect as shown here can be found in relation to the $R C$ harvesting circuit where it is important to see the characteristic equation comparison between voltage type and charge type systems. Here, the piezoelectric unimorph with tip mass offset can be formulated using the extended Hamiltonian principle to give,

$$
\left.\begin{array}{l}
\int_{t_{1}}^{t_{2}} \delta\left(L_{a}+W_{f}\right) d t=0
\end{array}\right\} \begin{gathered}
L_{a} \in\{K E, P E, W E\} \\
W_{f} \in\{W F\}
\end{gathered} \quad \text { or }
$$

After substituting Eq. (15) into Eq. (49) and further using the expression $\left(P E=P E^{(1)}+P E^{(2)}\right)$, Eq. (49) can be reformulated as,

$$
\int_{t_{l}}^{t_{2}}(\delta K E-\delta P E+\delta W E+\delta W F) \mathrm{d} t=0 .
$$

Note that each functional energy term of (50) can be formulated in (4)-(7). Also note that the harvesting circuit is excluded in (50) due to the charge type-based energy system from (10)-(11). However, the harvesting circuit system can be solved separately as shown in the next stage. The 
functional forms $L_{a}$ and $W_{f}$ from Hamiltonian's principle can be seen as the continuous differentiable functions of virtual displacement, electric displacement and charge for the whole systems that can be stated as,

$$
\begin{aligned}
L_{a} & =L_{a}\left(\dot{w}(x, t), \dot{w}(L, t), \frac{\partial \dot{w}(L, t)}{\partial x}, \frac{\partial^{2} w(x, t)}{\partial x^{2}}, E_{3}^{(2)}(z, t)\right), \\
W_{f} & =W_{f}\left(w(x, t), \frac{\partial w(L, t)}{\partial x}, w(L, t), v(t)\right) .
\end{aligned}
$$

Eqs. (51a)-(51b) can be further formulated using total differential equations as,

$$
\begin{aligned}
\delta L_{a}= & \frac{\partial L_{a}}{\partial \dot{w}(x, t)} \delta \dot{w}(x, t)+\frac{\partial L_{a}}{\partial \dot{w}(L, t)} \delta \dot{w}(L, t)+\frac{\partial L_{a}}{\partial\left(\frac{\partial \dot{w}}{\partial x}(L, t)\right)} \delta\left(\frac{\partial \dot{w}}{\partial x}(L, t)\right) \\
& +\frac{\partial L_{a}}{\partial E_{3}^{(2)}(z, t)} \delta E_{3}^{(2)}(z, t)+\frac{\partial L_{a}}{\partial\left(\frac{\partial^{2} w(x, t)}{\partial x^{2}}\right)} \delta\left(\frac{\partial^{2} w(x, t)}{\partial x^{2}}\right), \\
\delta W_{f}= & \frac{\partial W_{f}}{\partial w(x, t)} \delta w(x, t)+\frac{\partial W_{f}}{\partial\left(\frac{\partial w}{\partial x}(L, t)\right)} \delta\left(\frac{\partial w}{\partial x}(L, t)\right) \\
& +\frac{\partial W_{f}}{\partial w(L)} \delta w(L, t)+\frac{\partial W_{f}}{\partial v(t)} \delta v(t) .
\end{aligned}
$$

Corresponding with (52), Eq. (50) can be further formulated using the variational principle to give the electromechanical closed-form boundary value equation,

$$
\begin{aligned}
& \int_{t_{l}}^{t_{2}}\left[\int_{0}^{L}\left\{-I_{0} \ddot{w}(x, t)-I_{0} \ddot{w}_{\text {base }}(t)-C_{t} \frac{\partial^{2}}{\partial x^{2}}\left(\frac{\partial^{2} w(x, t)}{\partial x^{2}}\right)\right\} \delta w(x, t) \mathrm{d} x\right. \\
& -\left\{x_{c} I_{0}^{t i p} \frac{\partial \ddot{w}(L, t)}{\partial x}+I_{0}^{t i p} \ddot{w}(L, t)+I_{0}^{t i p} \ddot{w}_{\text {base }}\right\} \delta w_{\text {rel }}(L, t) \\
& +\left.C_{t} \frac{\partial}{\partial x}\left(\frac{\partial^{2} w(x, t)}{\partial x^{2}}\right) \delta w(x, t)\right|_{0} ^{L}-\left\{I_{2}^{t i p} \frac{\partial \ddot{w}(L, t)}{\partial x}+x_{c} I_{0}^{(t i p) \ddot{w}_{\text {base }}}\right. \\
& \left.+x_{c} I_{0}^{t i p} \ddot{w}(L, t)\right\} \delta \frac{\partial w(L, t)}{\partial x}-\left.\left\{\sigma v(t)+C_{t} \frac{\partial^{2} w(x, t)}{\partial x^{2}}\right\} \delta \frac{\partial w(x, t)}{\partial x}\right|_{0} ^{L} \\
& -\left\{\begin{array}{l}
\left.\left.\int_{0}^{L} \sigma \frac{\partial^{2} w(x, t)}{\partial x^{2}} \mathrm{~d} x-C_{v} v(t)-q_{1}(t)\right\} \delta v(t)\right] \mathrm{d} t=0 .
\end{array}\right.
\end{aligned}
$$

Note that some parameters from (53) can be found in Appendices A, B and C. Other parameters of mass moment of inertias can be seen in [20]. The reduced equation can be simplified using duBois-Reymond's theorem for each virtual displacement field. The first constitutive electromechanical damped dynamic equation can be formulated as,

$$
\delta w(x, t): I_{0} \ddot{w}(x, t)+I_{0} \ddot{w}_{\text {base }}(t)+C_{t} \frac{\partial^{2}}{\partial x^{2}}\left(\frac{\partial^{2} w(x, t)}{\partial x^{2}}\right)=0 .
$$

The second constitutive electromechanical dynamic equation can be formulated as,

$$
\delta v(t):-\int_{0}^{L} \sigma \frac{\partial^{2} w(x, t)}{\partial x^{2}} \mathrm{~d} x+C_{v} v(t)+q_{1}(t)=0 .
$$

Differentiating (55) with respect to time gives,

$$
-\int_{0}^{L} \sigma \frac{\partial^{2} \dot{w}(x, t)}{\partial x^{2}} \mathrm{~d} x+C_{v} \dot{v}(t)+i_{1}(t)=0 .
$$

Applying KCL for harvesting circuit in Fig. 1 gives,

$$
i_{1}=i_{2}+i_{3} \text {. }
$$

In the non-rectified harvesting circuit using (57), the parallel $R_{d} C_{d}$ circuit can be solved to give, 


$$
i_{1}=C_{d} \dot{v}(t)+\frac{v(t)}{R_{d}} .
$$

Eq. (56) can be further formulated using (58) to give,

$$
-\int_{0}^{L} \sigma \frac{\partial^{2} \dot{w}(x, t)}{\partial x^{2}} \mathrm{~d} x+\left(C_{v}+C_{d}\right) \dot{v}(t)+\frac{v(t)}{R_{d}}=0 .
$$

The boundary conditions can also be reduced to give,

$$
\begin{gathered}
w(0, t)=0 \quad, \frac{\partial w(0, t)}{\partial x}=0, \\
\delta w(L, t): x_{c} I_{0}^{t i p} \frac{\partial \ddot{w}(L, t)}{\partial x}+I_{0}^{t i p} \ddot{w}(L, t)+I_{0}^{t i p} \ddot{w}_{\text {base }}-C_{t} \frac{\partial}{\partial x}\left(\frac{\partial^{2} w(L, t)}{\partial x^{2}}\right)=0, \\
\delta \frac{\partial w(L, t)}{\partial x}: x_{c} I_{0}^{t i p} \ddot{w}(L, t)+x_{c} I_{0}^{t i p} \ddot{w}_{b a s e}+I_{2}^{t i p} \frac{\partial \ddot{w}(L, t)}{\partial x}+C_{t} \frac{\partial^{2} w(L, t)}{\partial x^{2}}+\sigma v(t)=0 .
\end{gathered}
$$

Applying (30) into (54) and (59)-(60), the results of which can be simplified to formulate the normalised closed-form electromechanical transverse dynamic equations with input base excitation, giving,

$$
\begin{gathered}
\ddot{w}_{r}(t)+2 \zeta_{r} \omega_{r} \dot{w}_{r}(t)+\omega_{r}{ }^{2} w(t)+P_{r} v(t)=-Q_{r} \ddot{w}_{\text {base }}(t), \\
\sum_{r=1}^{\infty} \hat{P}_{r}^{(w)} \dot{w}_{r}(t)+\left(C_{v}+C_{d}\right) \dot{v}(t)+\frac{v(t)}{R_{d}}=0 .
\end{gathered}
$$

The parameters $P_{r}, \hat{P}_{r}, P_{D}$, and $Q_{r}$ can be reduced as,

$$
\begin{gathered}
P_{r}=\sigma \frac{\mathrm{d} \hat{W}_{r}}{\mathrm{~d} x}(L), \sum_{r=1}^{\infty} \hat{P}_{r}=-\sum_{r=1}^{\infty} \int_{0}^{L} \sigma \frac{\mathrm{d}^{2} \hat{W}_{r}(x)}{\mathrm{d} x^{2}} \mathrm{~d} x, \\
Q_{r}=\int_{0}^{L} I_{0} \hat{W}_{r}(x) \mathrm{d} x+I_{0}^{t i p} \hat{W}_{r}(L)+x_{c} I_{0}^{t i p} \frac{\mathrm{d} \hat{W}_{r}(L)}{\mathrm{d} x} .
\end{gathered}
$$

Voltage FRFs across the resistor or capacitor can be formulated as,

$$
\frac{v(j \omega)}{-\omega^{2} w_{\text {base }} e^{j \omega t}}=\frac{\sum_{r=1}^{\infty} \frac{j \omega \hat{P}_{r} Q_{r}}{\omega_{r}{ }^{2}-\omega^{2}+j 2 \zeta_{r} \omega_{r} \omega}}{j \omega\left(C_{v}+C_{d}\right)+\frac{1}{R_{d}}-\sum_{r=1}^{\infty} \frac{j \omega \hat{P}_{r} P_{r}}{\omega_{r}^{2}-\omega^{2}+j 2 \zeta_{r} \omega_{r} \omega}} .
$$

The multi-mode electric current FRFs across the load resistance can be formulated as,

$$
\frac{i_{3}(j \omega)}{-\omega^{2} w_{\text {base }} e^{j \omega t}}=\frac{1}{R_{d}} \frac{v(j \omega)}{-\omega^{2} w_{\text {base }} e^{j \omega t}} .
$$

Power FRFs across the resistor and capacitor can be formulated respectively as,

$$
\begin{aligned}
& \frac{P_{\text {Cap }}(j \omega)}{\left(-\omega^{2} w_{\text {base }} e^{j \omega t}\right)^{2}}=j \omega C_{d}\left(\frac{v(j \omega)}{-\omega^{2} w_{\text {base }} e^{j \omega t}}\right)^{2}, \\
& \frac{P_{\text {Res }}(j \omega)}{\left(-\omega^{2} w_{\text {base }} e^{j \omega t}\right)^{2}}=\frac{1}{R_{d}}\left(\frac{v(j \omega)}{-\omega^{2} w_{\text {base }} e^{j \omega t}}\right)^{2} .
\end{aligned}
$$

\section{Result and discussion}

In this section, the intrinsic electromechanical dynamic behaviors of the piezoelectric power harvesting were discussed using the FRFs and time waveform DC output responses. The material properties of the piezoelectric unimorph are given in Table 1. The piezoelectric material used here was made from PZT PSI-5A4E. The geometrical structure with tip mass offset under input base excitation of $1 \mathrm{~m} / \mathrm{s}^{2}$ as shown in Fig. 1 has the length $L=60 \mathrm{~mm}$, and width $b=6 \mathrm{~mm}$ with the substructure (brass) thickness $h^{(1)}=0.5 \mathrm{~mm}$ and the piezoelectric thickness $h^{(2)}=0.127 \mathrm{~mm}$. The dimensions of the tip mass offset $l_{t}, h_{t}$ and $b$ (width) were set to $15 \mathrm{~mm}, 10 \mathrm{~mm}$ and $6 \mathrm{~mm}$, respectively. 
Table 1. Material properties of the piezoelectric bimorph

\begin{tabular}{lll}
\hline Material properties & Piezoelectric layers & Brass \\
\hline Young's modulus, $\bar{c}_{11}(\mathrm{GPa})$ & 66 & 105 \\
Density, $\rho\left(\mathrm{kg} / \mathrm{m}^{3}\right)$ & 7800 & 9000 \\
Piezoelectric constant, $d_{31}(\mathrm{pm} / \mathrm{V})$ & -190 & - \\
Permittivity, $\varepsilon_{33}^{T}(\mathrm{~F} / \mathrm{m})$ & $1800 \varepsilon_{o}$ & - \\
Permittivity of free space, $\varepsilon_{\mathrm{o}}(\mathrm{pF} / \mathrm{m})$ & 8.854 & - \\
\hline
\end{tabular}
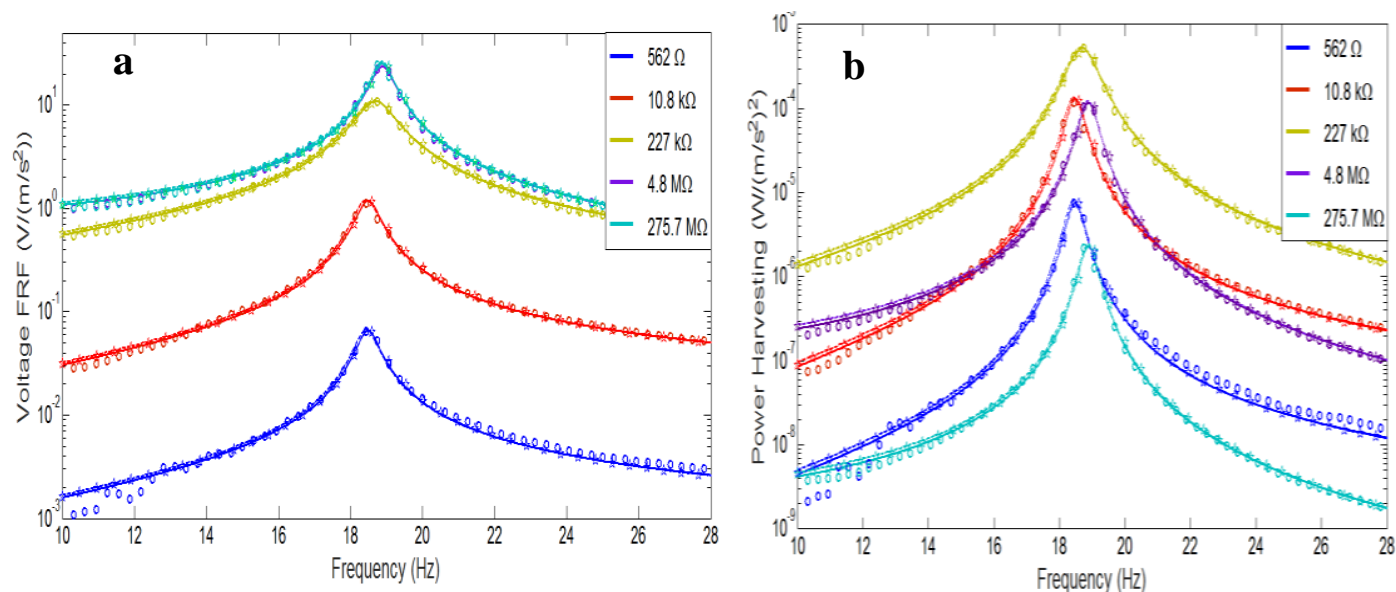

Fig. 3 Electromechanical FRFs under variable load resistances with numerical (solid lines), analytical voltage type (dash lines), analytical charge type (pentagram) and experimental results (round dot): (a) voltage and (b) power harvesting across load resistance
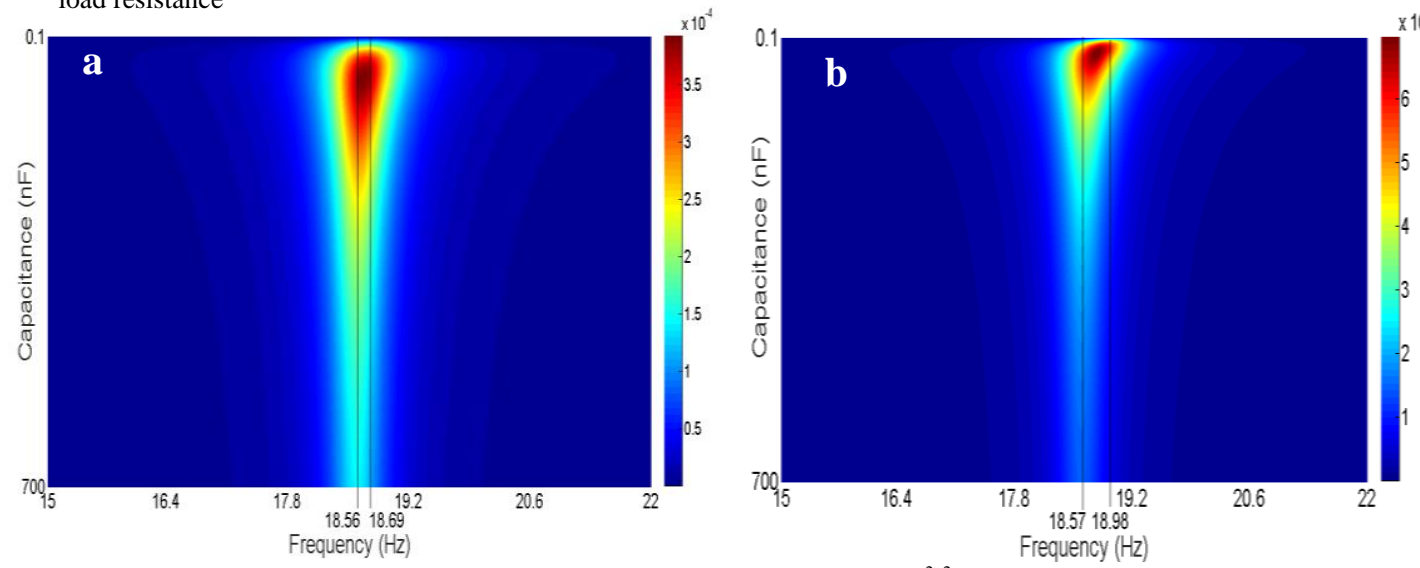

Fig. 4 Power harvesting contour electromechanical dynamic responses $\left(\mathrm{W} /\left(\mathrm{m} / \mathrm{s}^{2}\right)^{2}\right)$ : (a) capacitance variation with fixed load resistance of $155 \mathrm{k} \Omega$, (b) capacitance variation with fixed load resistance of $4.8 \mathrm{M} \Omega$
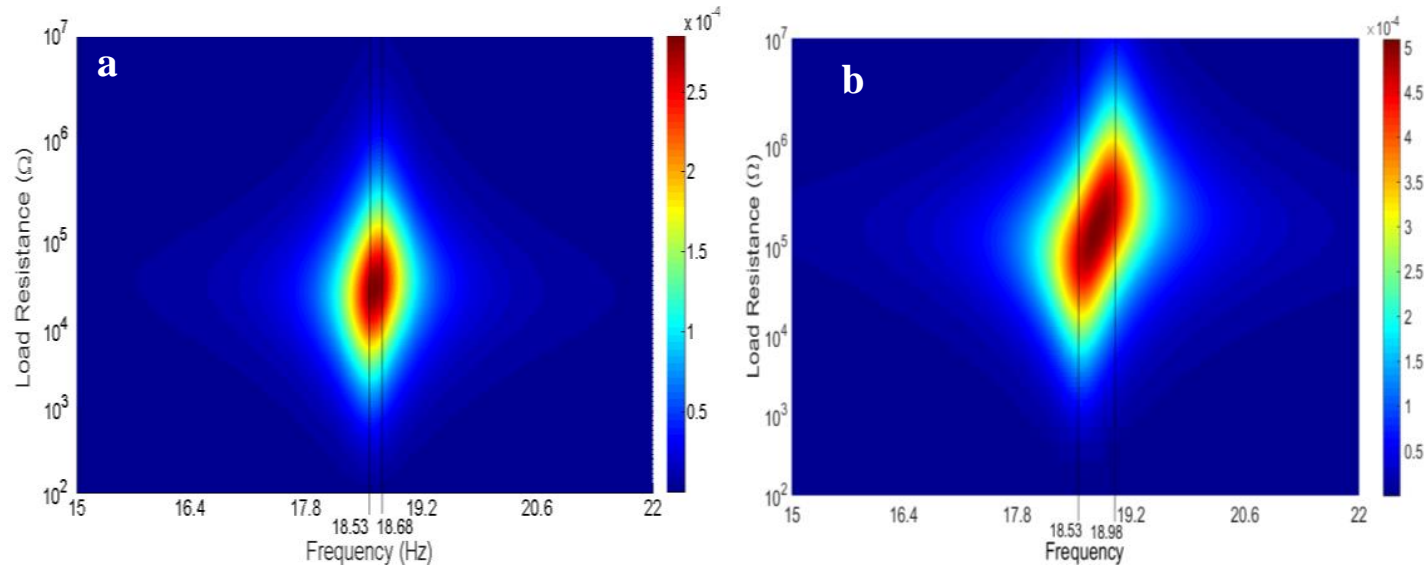

Fig. 5 Power harvesting contour electromechanical dynamic responses $\left(\mathrm{W} /\left(\mathrm{m} / \mathrm{s}^{2}\right)^{2}\right)$ : (a) load resistance variation with fixed capacitance of $0.1 \mu \mathrm{F}$, (b) load resistance variation with fixed capacitance of $1 \mathrm{pF}$ 

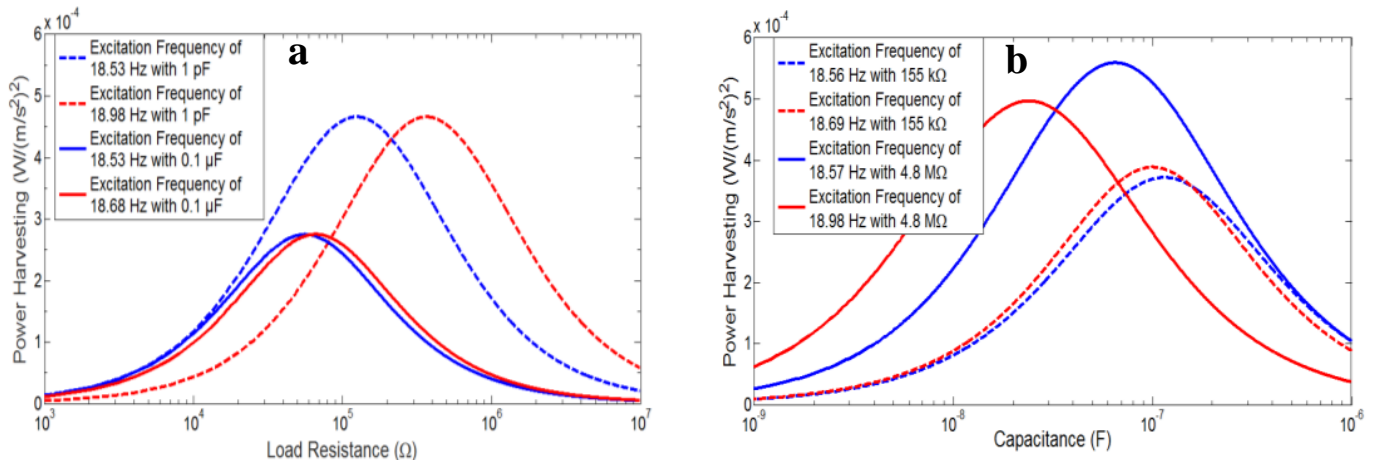

Fig. 6 Power harvesting under excitation frequencies $\left(\mathrm{W} /\left(\mathrm{m} / \mathrm{s}^{2}\right)^{2}\right)$ : (a) load resistance variation with fixed capacitances of $1 \mathrm{pF}$ and $0.1 \mu \mathrm{F}$, (b) capacitance variation with fixed load resistances of $155 \mathrm{k} \Omega$ and $4.8 \mathrm{M} \Omega$

In Fig. 3, the trends of voltage and power harvesting FRFs with variable load resistance using four different methods show excellent agreement under open circuit capacitive load (very low capacitive). The results showed in Fig. 3 includes the comparisons between analytical charge and voltage type systems (Sections 4.1 and 4.2), electromechanical finite element modelling [20] and experimental results [16]. However, the significant difference between previous works and the present work is that the two complete theoretical methods (analytical charge and voltage types) as shown at Sections 4.1 and 4.2 were formulated and discussed by including $R C$ circuit and standard DC interface circuit, and more importantly, scrutinizing the intrinsic physical behaviours. Therefore, for validating the previous works with resistive load [16], [20], the two theoretical studies must be set to the capacitance with very low value $(1 \mathrm{pF})$ so as to achieve sufficient virtually open circuit conditions, since the previous works show the power harvesting with only resistive load. Note that since the analytical charge type system in Fig. 3 shows good agreement with other methods, the forthcoming figures only show the case study results from the analytical charge type modelling.

Further technical aspects of the power harvesting behaviour across capacitive load can be seen in Fig. 4. It is clearly seen that trends of power output FRFs across the variable capacitive load gives the frequency shift using certain fixed values of resistances. In this stage, the system response shows the shifting resonances due to, not only the variable capacitance, but also the particular values of load resistance resulting in shifting amplitudes. This means that for the chosen load resistance parameters, the shifting resonances from virtually short to open circuit conditions of the capacitive loads can change the trend of the system response resulting in changing amplitude levels. At the lower region of capacitance (dark red colour), the system response reaches the maximum amplitude level which is away from short and open circuit conditions. It is obvious to see that the resonance responses under virtually short and open circuit conditions of the capacitive loads only give the lowest amplitude levels as shown in the two parallel lines from Figs. 4a and $4 \mathrm{~b}$. For example, virtually short and open circuit conditions for capacitive loads in Fig. $4 \mathrm{~b}$ occur for the resonances of $18.57 \mathrm{~Hz}$ and $18.98 \mathrm{~Hz}$, respectively.

Slightly different behaviour can be seen in Fig. 5, where the shift of resonance of the power output across variable load resistance can be seen with the use of the fixed capacitance of $0.1 \mu \mathrm{F}$ and even further wider resonance shift when using the fixed capacitance of $1 \mathrm{pF}$. With the fixed capacitance of $0.1 \mu \mathrm{F}$, the short and open circuit load resistances provide the resonance shift from $18.53 \mathrm{~Hz}$ to $18.68 \mathrm{~Hz}$, respectively. For the fixed capacitance of $1 \mathrm{pF}$, the short and the open circuit load resistances provide the resonance shift from $18.53 \mathrm{~Hz}$ to $18.98 \mathrm{~Hz}$, respectively. The wider resonance shift occurs due to the capacitance of $1 \mathrm{pF}$ virtually approaching open circuit condition. It is obvious to see that the power output appears to shift due to the contribution of not only load resistance, but also capacitance changes. It is important to note here that decreasing the capacitance values can result in increasing the power output across load resistance. Upon reaching the maximum power level, further reducing the capacitance value has no effect on the power. However, it should be noted that for the time waveform response, using very small value of capacitance such as $1 \mathrm{pF}$ can affect the large ripple voltage giving ineffective charging process of the capacitor where the discharging process will increase. Note that the system response behaviour in Fig. 5b shows similar trend with Fig. 3b. For specific cases, Figs. 4 and 5 can also be visualized by only focusing on the excitation frequencies with certain fixed electrical parameter values that represent the resonances approaching the short and open circuit conditions as shown in Fig. 6. As can be seen in Fig. 6a, the maximum power outputs at those resonances with fixed capacitances of $1 \mathrm{pF}$ and $0.1 \mu \mathrm{F}$ can be found at the regions away from short and open circuit load resistances. The 
power output with the capacitance of $1 \mathrm{pF}$ gives higher value compared with the use of $0.1 \mu \mathrm{F}$. However, as mentioned previously, the ripple voltage will occur when using very small capacitance value. In similar fashion, the trends of power output in Fig. $6 \mathrm{~b}$ with the capacitances away from short and open circuit values also show the maximum amplitudes using the fixed load resistances of $155 \mathrm{k} \Omega$ and $4.8 \mathrm{M} \Omega$.

For the DC time waveform response at the frequency of $18.6 \mathrm{~Hz}$, the trend of the DC voltage through the full-wave bridge rectifier (Fig.7a) can be seen through the process of the positive or negative half-cycles of the AC signal becoming the positive ripple signal due to the diode pairs (D1 and D2) and (D3 and D4) interchangeably turning to conduct. With the smoothing capacitor connection, the ripple of DC voltage output (Fig. 7a) reduces allowing the process of charging and discharging for every half-cycle. As a result, capturing the DC current through the capacitor can only be seen during the process of charging (Fig. 7b). Note that maintaining the DC voltage level depends not only on the chosen capacitor value but also on the load resistance value as shown in the example in Fig. 7. It should be noted that the piezoelectric component with its inherent capacitance and electromechanical coupling, including the dynamic system, also affects the DC time waveform signal that should be taken into consideration. Unlike the action of the DC current through the capacitor, the DC current across the resistor can be captured so as to maintain and take the electrical energy from the capacitor. Moreover, the process of capturing the DC power across the load resistance using different frequencies can be visualized in more detail in the next stage.
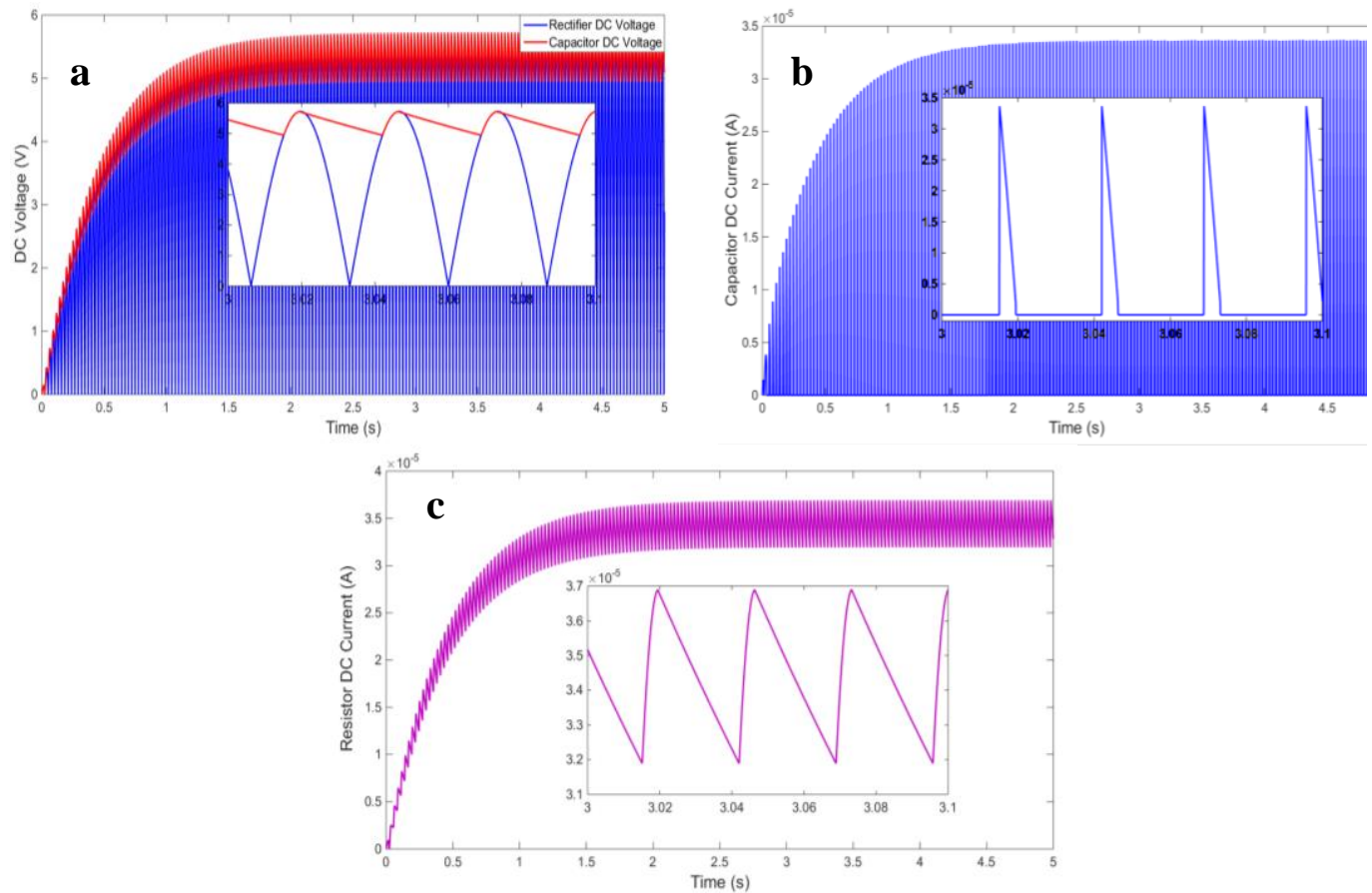

Fig. 7 Time waveform signal based on load resistance of $155 \mathrm{k} \Omega$ with fixed capacitance of $0.1 \mu \mathrm{F}$ : (a) DC voltage, (b) DC current through capacitor and (c) DC current through resistor

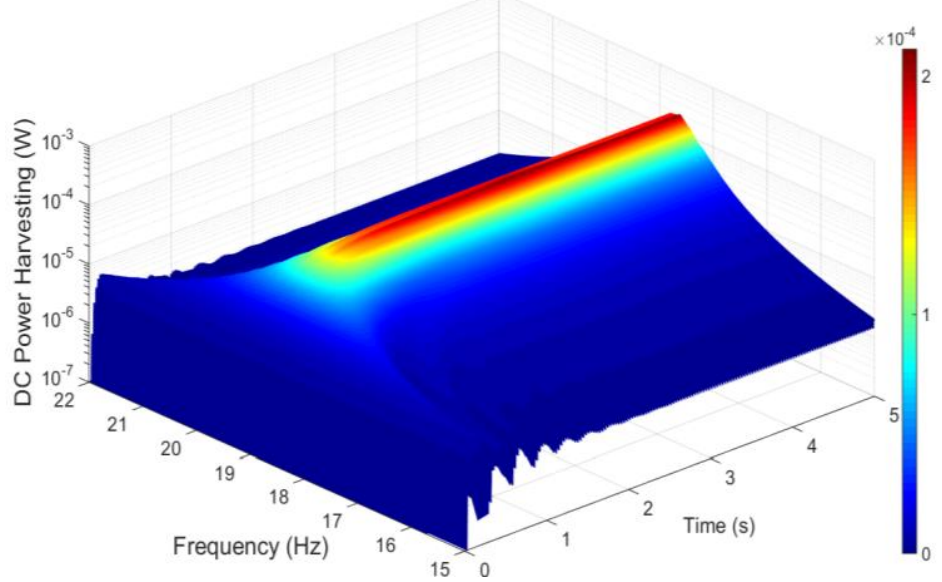

Fig. 8 3D spectrogram visualisation of electromechanical power harvesting across load resistance of $155 \mathrm{k} \Omega$ with fixed capacitance of $0.1 \mu \mathrm{F}$ 
As shown in Fig. 8, the 3D spectrogram of the DC power harvesting across load resistance shows the behaviour of the frequency spectrum of the system as a function of time. It clearly shows that the resonance region provides the peak amplitude with constant value essentially after 1 second. At the off-resonance region, the fluctuated waveform before 1 second due to the initial transient appears to be more pronounced. After that the waveform of the power amplitude tends to be stable with constant behaviour. The importance of the $3 \mathrm{D}$ spectrogram in the power harvesting scheme can provide the useful information of the time waveform interval of the system responses during operation of the different frequency or vice versa. Note that visualisation of the 3D spectrogram can only be shown using the fixed parameter values of the RC circuit and physical system of the piezoelectric power harvesting device. For example, if the load resistance changes, the 3D spectrogram shows similar behaviour to that shown previously. However, the resonance frequency region will be different along the time axis depending on the values of load resistance (from short to open circuit load resistances). As a result, the collection of each spectrogram using variable load resistance will show exactly the same frequency shift and amplitude results as shown in Fig. 5.

\section{Conclusion}

This paper has presented the intrinsic electromechanical system formulations of piezoelectric power harvesters. The important aspect of the formulations is that it provides fundamental techniques for exploring the piezoelectric structure coupled with the AC-DC circuit interface. The vast majority of the previous published piezoelectric power harvesting papers have used the constitutive piezoelectric equations reduced from the electrical enthalpy energy as a basis to develop their further analytical studies in many different applications. Nevertheless, the previous works have not explored distinct electromechanical equations in relations to the piezoelectric system and the harvesting circuit that can be used to formulate frequency and time waveform responses. Moreover, it is noted here that the use of the analytical charge type-based Helmholtz free energy and voltage type-based electrical enthalpy energy reduced from the variational principle provides insight into the technical concepts for formulating the frequency and time waveform responses. The analytical charge type modelling provides more compact equations compared with the analytical voltage type equations representing direct relevancy for deriving simultaneous formulations of the piezoelectric structure and electronic system using functional energy forms from the extended Hamiltonian principle. As a result, the two analytical methods have shown good agreement with the established electromechanical finite element and experimental studies. Moreover, the power harvesting system responses using variable load resistance and capacitance have been explored in order to identify the behaviour of the shifting frequency and amplitude. The DC time waveform outputs provide the process of capturing the voltage signal behaviours through the rectifier and capacitor including the current through the capacitor and power harvesting across the load resistance. Detailed visualisation for the power time waveform signal with different operating frequencies has also been provided using the spectrogram. Overall, the major contribution of the paper has shown the interconnection between the fundamental physical parameters of charge and voltage type equations and electrical systems using the variational principle so as to formulate the power harvesting system responses where the techniques can be extended in many different applications.

\section{A Appendix: General Stiffness Coefficients for the Smart Structure}

The total transverse stiffness coefficient for triple layers can be formulated as,

$$
C_{t}=\frac{1}{3}\left(\sum_{i=1}^{m} \bar{c}_{g}^{(i)} b^{(i)}\left(\sum_{j=i}^{m} h^{(j)}-z_{n}\right)^{3}-\sum_{i=1}^{m-1} \bar{c}_{g}^{(i)} b^{(i)}\left(\sum_{j=i+1}^{m} h^{(j)}-z_{n}\right)^{3}+\bar{c}_{g}^{(m)} b^{(m)} z_{n}{ }^{3}\right) .
$$

Note that the general parameter $\bar{c}_{g} \in\left\{\bar{c}_{D}, \bar{c}_{11}\right\}$ indicates elastic stiffness where $\bar{c}_{D}$ and $\bar{c}_{11}$ are for charge and voltage types, respectively. Also note that the substructure layer for charge type form $\bar{c}_{D}^{(1)}=\bar{c}_{11}^{(1)}$ is for non-piezoelectric material and the neutral axis $z_{n}$ can be seen in Appendix B.

Parameter $b$ is the width of the interlayer and term $m$ is the number of layers. 


\section{B Appendix: General Neutral Axis of the Smart Structure}

In Fig. 1, the location of the asymmetric neutral axis measured from the $x$-axis to the top surface of the piezoelectric layer can be determined using the resultant force balance in the cross-section of the unimorph structure to give,

$$
z_{n}=\frac{\sum_{i=1}^{m} \bar{c}_{g}^{(i)} h^{(i)^{2}} b^{(i)}+2 \sum_{i=1}^{m-1} \bar{c}_{g}^{(i)} h^{(i)} b^{(i)} \sum_{j=i+1}^{m} h^{(j)}}{2 \sum_{i=1}^{m} \bar{c}_{g}^{(i)} h^{(i)} b^{(i)}} .
$$

\section{Appendix: General Transverse Piezoelectric Coupling Coefficient and Piezoelectric Capacitance}

The transverse piezoelectric coupling for charge and voltage types can be formulated, respectively as,

$$
\begin{gathered}
\eta=\frac{g_{31}\left(2 z_{n} h^{(2)}-h^{(2)^{2}}\right)}{2 L}, \\
\sigma=-\frac{e_{31} b^{(2)}\left(2 z_{n} h^{(2)}-h^{(2)^{2}}\right)}{2 h^{(2)}} .
\end{gathered}
$$

The inherent piezoelectric capacitance for both charge and voltage type forms can be stated, respectively as,

$$
C_{v}=\frac{\varepsilon_{33}^{S} b^{(2)} L}{h^{(2)}} .
$$

\section{Reference}

1. Irschik, H., Krommer, M., Belyaev, A.K., Schlacher, A.K.: Shaping of piezoelectric sensors/actuators for vibrations of slender beams: coupled theory and inappropriate shape functions. J. Intell. Mater. Syst. Struct. 9, 546-554 (1998)

2. Krommer, M., Zellhofer, M., Heilbrunner, K.-H.: Strain-type sensor networks for structural monitoring of beam-type structures. J. Intell. Mater. Syst. Struct. 20, 1875-1888 (2003)

3. Irschik, H., Pichler, U.: Dynamic shape control of solids and structures by thermal expansion strains. J. Therm. Stresses, 24, 565-576 (2001)

4. Tzou, H.S., Tseng, C.I.: Distributed vibration control and identification of coupled elastic/piezoelectric systems: finite element formulation and applications. Mech. Syst. Signal Process, 5 (3), 215-231 (1991)

5 Lam, K.Y., Peng, X.Q., Liu, G.R., Reddy, J.N.: A finite-element model for piezoelectric composite laminates. Smart Mater. Struct. 6, 583-591 (1997)

6. Krommer, M., Irschik, H.: Sensor and actuator design for displacement control of continuous systems. Smart Struct. Syst. 3, 147-172 (2007)

7. Niederberger, D., and Morari, M.: An autonomous shunt circuit for vibration damping. Smart Mater. Struct. 15 359-364 (2006)

8. Vasques, C.M.A.: Improved passive shunt vibration control of smart piezo-elastic beams using modal piezoelectric transducers with shaped electrodes. Smart Mater. Struct. 21, 125003 (2012)

9. Roundy, S., Wright, P.K: A piezoelectric vibration based generator for wireless electronics. Smart Mater. Struct., 18, 1131-1142 (2004)

10. Liao, Y., Sodano, H.: Modeling and comparison of bimorph power harvesters with piezoelectric elements connected in parallel and series. J. Intell. Mater. Syst. Struct. 21, 14959,2010

11. Kim, M., Hoegen, M., Dugundji, J., Wardle, B.L.: Modeling and experimental verification of proof mass effects on vibration energy harvester performance, Smart Mater. Struct. 19 045023 (2010)

12. Erturk, A.: Assumed-modes modeling of piezoelectric energy harvesters: Euler-Bernoulli, Rayleigh, and Timoshenko models with axial deformations. Comp. Struct. 106-107, 214-27, (2012) 
13. Lumentut, M.F., Howard, I.M.: Analytical and experimental comparisons of electromechanical vibration response of a piezoelectric bimorph beam for power harvesting. Mech. Syst. Signal Process. 36, 66-86 (2013)

14. Wang, H., Meng, Q.: Analytical modeling and experimental verification of vibration-based piezoelectric bimorph beam with a tip-mass for power harvesting. Mech. Syst. Signal Process. 36,193-209 (2013)

15. Dalzell, $\mathrm{P}$ and Bonello, P.:Analysis of an energy harvesting piezoelectric beam with energy storage circuit. Smart Mater.Struct. 21, 105029 (2012)

16. Lumentut, M.F., Howard, I.M.: Parametric design-based modal damped vibrational piezoelectric energy harvesters with arbitrary proof mass offset: Numerical and analytical validations. Mech. Syst. Signal Proc. 68, 562-586 (2015)

17. Elvin, N.G., A.A. Elvin, A.A.: Coupled finite element-circuit simulation model for analyzing piezoelectric energy generators. J. Intell. Mater. Syst. Struct. 20 587-595 (2009)

18. Yang, Y., Tang, L.: Equivalent circuit modeling of piezoelectric energy harvesters. J. Intell. Mater. Syst. Struct. 20, 2223-2235 (2009)

19. Wu, P.H., Shu, Y.C.: Finite element modeling of electrically rectified piezoelectric energy harvesters. Smart Mater. Struct. 24, 094008 (2015)

20. Lumentut, M.F., Howard, I.M.: Electromechanical finite element modelling for dynamic analysis of a cantilevered piezoelectric energy harvester with tip mass offset. Smart Mater. Struct. 23, 095037 (2014)

21. Yang, Z.T., Yang, J.S.: Connected vibrating piezoelectric bimorph beams as a wide-band piezoelectric power harvester. J. Intell. Mater. Syst. Struct. 20 (5) 569-574 (2009)

22. Lumentut, M.F., Francis, L.A., Howard, I.M.: Analytical techniques for broadband multielectromechanical piezoelectric bimorph beams with multifrequency power harvesting. IEEE Trans. Ultrason. Ferroelectr. Freq. Control. (59) 1555-1568 (2012)

23. Lien, I.C., Shu, Y.C.: Array of piezoelectric energy harvesting by the equivalent impedance approach. Smart Mater. Struct. 21, 082001 (2012)

24. Lin, H.C., Wu, P.H., Lien, I.C., Shu, Y.C.: Analysis of an array of piezoelectric energy harvesters connected in series. Smart Mater. Struct. 22, 094026 (2013)

25. Xiong, X., Oyadiji, S.O.: Modal optimization of doubly clamped base-excited multilayer broadband vibration energy harvesters. J. Intel. Mat. Syst. Struct. doi: $10.1177 / 1045389 X 14551433$ (2014)

26. Zhang, H., Afzalul, K.: Design and analysis of a connected broadband multi-piezoelectricbimorph-beam energy harvester. IEEE Trans. Ultrason. Ferroelectr. Freq.Control. 61 10161023 (2014)

27. Lefeuvre E, Badel A, Richard C, Petit L, Guyomar D.: A comparison between several vibration-powered piezoelectric generators for standalone systems. Sens Actuat A-Phys. 126 405-416 (2006)

28. Tang, L., Yang, Y.: Analysis of synchronized charge extraction for piezoelectric energy Harvesting. Smart Mater. Struct. 20, 085022 (2011)

29. Guyomar, D., Badel, A., Lefeuvre, E., Richard, C.: Toward energy harvesting using active materials and conversion improvement by nonlinear processing. IEEE Trans. Ultrason. Ferroelectr. Freq. Control. 52, 584-95 (2005)

30. Shu, I.C., Lien, I.C., Wu, W.J.: An improved analysis of the SSHI interface in piezoelectric energy harvesting. Smart Mater. Struct. 16, 2253-2264 (2007)

31. Lallart M, Guyomar D.: An optimized self-powered switching circuit for nonlinear energy harvesting with low voltage output. Smart Mater Struct 17, 035030 (2008)

32. Lumentut, M.F., Howard, I.M.: Effect of shunted piezoelectric control for tuning piezoelectric power harvesting system responses-Analytical techniques. Smart Mater. Struct. 24(10), 105029 (2015)

33. Standards Committee of the IEEE Ultrasonics, Ferroelectrics, and Frequency Control Society 1987 IEEE Standard on Piezoelectricity (New York: IEEE)

34. Tiersten, H.F., Linear Piezoelectric Plate Vibrations (New York: Plenum, 1969)

35. Nye, J.F., Physical Properties of Crystals: their Representation by Tensors and Matrices (Oxford: Clarendon, 1984)

36. Ikeda, T., Fundamentals of Piezoelectricity (New York: Oxford University Press, 1990)

37. Tichý, J., Erhart, J., Kittinger, E., Prívratská, J., Fundamentals of piezoelectric sensorics, (Berlin: Springer-Verlag, 2010) 